\title{
How "malignant" is the neuroleptic malignant syndrome?
}

\author{
In early mild cases it may not be malignant at all
}

Although cases of acute fatal psychoses associated with fever and neuromuscular disturbance have been reported sporadically since the nineteenth century ${ }^{1}$ and the propensity for neuroleptic drugs to disrupt neuromuscular function has been described as their use has grown, the entity of the "neuroleptic malignant syndrome" is a comparatively recent phenomenon, mentioned in standard British psychiatric textbooks only for the past decade. In its brief lifespan it has generated considerable controversy with regard to its prevalence, diagnostic criteria, and management.

A recent review estimated that the prevalence of the syndrome varied from $0.02 \%$ to $2 \%$ of admissions to acute psychiatric wards. ${ }^{2}$ Acute psychiatry is not the only domain of this disorder; cases have been described in patients with other conditions treated with neuroleptics and patients whose treatment with dopamine precursors has been stopped. All neuroleptics that are currently used have been incriminated. The mortality, estimated at $20 \%$ in the early 1980 s, seems to be falling, ${ }^{3}$ which may be ascribed to greater awareness of the condition and its earlier detection.

Other explanations may exist for the falling mortality and the 100 -fold variation in prevalence mentioned above. Although the neuroleptic malignant syndrome is described as having four classic signs (fever, rigidity, autonomic instability, and altered consciousness), no agreed criteria exist for the diagnosis of the syndrome in terms of severity or combination of these signs, and, as systematic searches for the syndrome have increased, milder or incomplete varieties (formes frustes) have been detected and included with the full blown cases. The neuroleptic malignant syndrome does not now seem quite as malignant as originally thought. Some authors believe that it is at one end of a range of effects induced by neuroleptics, such as parkinsonism or dystonia ${ }^{4}$; others that the term should be reserved for the full blown syndrome, ${ }^{5}$ which has the features of an idiosyncratic reaction more akin to malignant hyperpyrexia. The relation between the neuroleptic malignant syndrome and catatonia is also unclear. ${ }^{6}$

This debate has crucial implications for management. Consensus exists about managing the full blown condition: immediate cessation of neuroleptics, rehydration, and correction of fever and acidosis are vital. Electroconvulsive therapy and benzodiazepines during an episode are considered to be safe. Rechallenge with neuroleptics, the mainstay of treatment for psychosis, is probably safe, ${ }^{7}$ provided that a gap of two weeks is left between the resolution of the neuroleptic syndrome and the reintroduction of the drug.
This should be done cautiously, with careful monitoring of physical signs. Less agreement exists about the utility of bromocriptine and dantrolene; one review found that both, especially bromocriptine, shortened the time to the resolution of symptoms when compared with supportive measures alone. $^{8}$

But management of mild or early cases gives rise to debate: Withdrawing neuroleptics may delay resolution or even worsen the psychosis, and withdrawing them from every patient with fever and rigidity would be questionable, especially given the availability of beds for psychiatric patients. Alternatively, treating mild or early cases with anticholinergics alone might delay recognition of the full blown syndrome.

The waters are muddied still further by the use of creatine kinase to identify the syndrome. In most of the first described cases the patients had high concentrations of creatine kinase (over $1000 \mathrm{IU} / \mathrm{l}$ ), and measurement of this enzyme has since been used as a diagnostic test. In full blown cases large rises in creatine kinase concentration are the rule. But the muscle isoenzyme of creatine kinase may be raised by intramuscular injections, hyperactivity, and catatonia ${ }^{9}$ and in medically ill patients taking neurolpetics. ${ }^{10}$ Thus, it is not a specific indicator of the neuroleptic malignant syndrome and its importance in the early detection of the syndrome and other forms of neuromuscular disturbance remains doubtful.

Resolving the debate between those who believe that neuroleptic malignant syndrome is a rare idiosyncrasy and the advocates of a continuum between the early, milder forms and the full blown syndrome is impossible until a testable causal model emerges. Possibilities include malfunctioning central dopaminergic receptors, ${ }^{11}$ sympatheticomedullary system, ${ }^{12}$ or central $N$-methyl-D-aspartate-glutamate receptors. ${ }^{13}$ But a better understanding of the natural course of the formes frustes and the importance of raised creatine kinase concentrations in acute psychosis would also help. Meanwhile, the malignancy of the neuroleptic malignant syndrome remains debatable.

When faced with a patient taking neuroleptics who has a fever the doctor should always look for another somatic illness while bearing in mind the possibility of the syndrome. In one series of 34 cases of suspected neuroleptic malignant syndrome, 10 of them included other intercurrent febrile conditions. ${ }^{14}$ But themostpressing question for doctors dealingwith psychotic patients who might be developing the syndrome-when to stop neuroleptics-remains unanswered. One approach might 
be to draw up a severity scale with set treatments for each level. In one such management plan, stopping neuroleptics is not mandatory until fever exceeds a certain threshold. ${ }^{15} \mathrm{We}$ wait to see the results of such a plan but in the meantime should develop our own.

M F BRISTOW Lecturer

D KOHEN

Senior lecturer

Charing Cross and Westminster Medical School,

Charing Cross Hospital,

London W6 8RF

1 Kellam AMP. The neuroleptic malignant syndrome, so-called. Br f Psychiatry 1987;150:752-9.

2 Keck PE, McElroy SL, Pope HG. Neuroleptic malignant syndrome. Current opinion in psychiatry 1991;4:34-7.
3 Shalev A, Hermesh H, Munitz H. Mortality from neuroleptic malignant syndrome. f Clin Psychiatry 1989;50:18-25.

Reilly J, Crowe SF, Loyd JH. Neuroleptic toxicity syndromes: a clinical spectrum. Aust $\mathrm{NZ}$ Psychiatry 1991;25:499-505.

5 Adityanjee. The myth of elevated serum CPK and neuroleptic malignant syndrome. Br $\mathcal{F}$ Psychiatry 1991;158:706-7.

6 White DA. Catatonia and the NMS-a single entity? Br 7 Psychiatry 1992;161:558-60.

7 Rosebush PA, Stewart T, Gelenberg LAJ. Twenty neuroleptic rechallenges after neuroleptic malignant syndrome. F Clin Psychiatry 1989;50:295-8.

8 Rosenberg MR, Green M. Neuroleptic malignant syndrome. Arch Intern Med 1989;149:1927-31.

9 Craddock B, Craddock N, Milner G. CPK in NMS. Br f Psychiatry 1991;158:130.

10 O'Dwyer A, Sheppard $N$. The role of creatine kinase in the diagnosis of the neuroleptic malignant syndrome. Psychol Med 1993;23:323-6.

11 Nisijima $K$, Ishiguro T. Neuroleptic malignant syndrome. A study of CSF monoamine metabolism. Biol Psychiatry 1990;27:280-8.

12 Guerrera RJ, Romero JA. Sympathomedullary activity in the neuroleptic malignant syndrome. Biol Psychiatry 1992:32:334-43.

13 Weller M, Kornhuber J. A rationale for NMDA receptor antagonist therapy of the neuroleptic malignant syndrome. Med Hypotheses 1992;38:329-33.

4 Sewell DD, Jeste DV. Distinguishing NMS from NMS-like acute medical illnesses: a study of 34 cases. Foumal of Neuropsychiatry 1992;4:265-9.

15 Gratz SS, Levinson DF, Simpson GM. The treatment and management of neuroleptic malignant syndrome. Prog Neuropsycopharmacol Biol Psychiatry 1992;16:425-43.

\section{Human T cell leukaemia/lymphoma virus and blood donation}

\section{Routine screening of blood donors is not currently indicated in Britain}

On p 1235 Brennan and colleagues report that about one in 20000 blood donors in north London is positive for antibodies to human T cell leukaemia/lymphoma virus (HTLV). ${ }^{1}$ If this prevalence is typical of that in the rest of Britain they estimate that each year up to six people may be put at risk of developing disease associated with HTLV in their lifetime. The United States, Japan, and some European countries routinely screen blood for antibodies to HTLV; if Britain was to follow suit it would cost $£ 1.3 \mathrm{~m}$ to prevent each case of disease associated with HTLV acquired from transfusion.

HTLV type I (HTLV-I) was first isolated nearly 15 years ago from a patient with a variant of what is now accepted to be the adult $\mathrm{T}$ cell leukaemia/lymphoma syndrome. This syndrome was described in Japan before the discovery of HTLV-I and usually presents as an acute T cell leukaemia (or lymphoma, or both) with skin lesions, lymphadenopathy, hepatosplenomegaly, hypercalcaemia, and bone cysts. In the absence of an overt leukaemia the clinical picture often suggests sarcoidosis. It is now recognised that HTLV-I may be associated with a wide range of $T$ cell malignancies, including prodromal and chronic leukaemic states. In addition, HTLV-I is closely associated with tropical spastic paraparesis, a condition also known in Japan as HTLV-I associated myelopathy. The condition is similar to some presentations of multiple sclerosis but without the remissions or relapses. ${ }^{2}$

The pathogenesis of tropical spastic paraparesis associated with HTLV-I is of great interest as the virus does not seem to be directly responsible for the damaged neurological tissue. ${ }^{3}$ An intense lymphocytic infiltration round the demyelinated area strongly suggests an immunopathogenic mechanism of destruction. Other autoreactive conditions have also recently been recognised as being associated with HTLV-I infection, including arthritis and uveitis. ${ }^{4}$

The mechanisms whereby a "simple" retrovirus can induce such diverse clinical conditions after an incubation period of up to three or four decades are poorly understood. Unlike other oncogenic retroviruses, HTLV-I does not carry the cellular oncogene homologue; nor does it insert site specifically into the DNA and "turn on" oncogenes in cis (or interfere with suppressor or control genes). Instead, HTLV-I encodes a regulatory mechanism similar to that described for HIV where one of the proteins that normally regulates its own division can also turn on or activate cellular genes and their receptors, which may not necessarily be close to the site of integration of the provirus-that is, in trans. Among other genes, HTLV-I can activate the genes for T cell growth factor interleukin-2 and its receptor-a mechanism that allows the cell to stimulate itself autonomously. But this autonomous mechanism for continued cell activation is not the mechanism for inducing malignant change directly, although activating $T$ cells long term may make the cells more susceptible to second and third oncogenic events.

The mechanism whereby HTLV-I causes tropical spastic paraparesis also involves the activation of lymphocytes, presumably by a different mechanism as they do not contain the HTLV-I provirus. ${ }^{5-8}$ A genetic haplotype association has been described from Japan, and differences in the specificity of the cytotoxic $\mathrm{T}$ lymphocyte responses claimed between tropical spastic paraparesis and asymptomatic patients remain controversial. ${ }^{9}$

Abnormal proliferation and differentiation of $T$ cells in vitro, apparently sensitive to rapamycin but not to cyclosporin A, suggests a specific mechanism of cellular activation, ${ }^{10}$ perhaps at the cellular membrane level, by the HTLV-I envelope. Recent work suggests that the ability to infect antigen presenting cells, such as dendritic cells, may be important in this process. ${ }^{11}$

HTLV-II is a similar virus which is less closely linked to diseases yet is prevalent particularly among drug addicts in Europe and the United States. Early suggestions that it is linked to hairy cell leukaemia have not been substantiated. ${ }^{23}$

Infection with HTLV-I and HTLV-II is endemic in Japan and the Caribbean and occurs in discrete pockets throughout the rest of the world. The large Caribbean population in Britain accounts for its presence here. HTLV-I is less infectious than HIV, although it is spread through the same blood borne and sexual routes. It can also be spread by breast feeding. Knowledge of whether it is spreading in the community is therefore important. Early serological studies suggested that HTLV-I was present in the Caribbean community and only rarely (anecdotally) in the white population and that even British born children of parents infected with HTLV-I were unlikely to be infected. ${ }^{12}$ This has led to epidemiological studies suggesting that a cofactor not well represented in Britain is required for transmission. 